\title{
Evaluación del consumo de drogas ilícitas per cápita por epidemiología de alcantarilla: impacto en el medio ambiente y salud pública
}

\author{
Avaliação do consumo de drogas ilícitas per capita por epidemiologia de águas residuais: \\ impacto no meio ambiente e saúde pública
}
Evaluation of illicit drug consumption per capita by wastewater-based epidemiology: impact on the environment and public health

Aldo Pacheco Ferreira1*, María José Cruz-Hernández².

\begin{abstract}
RESUMEN
Objetivo: Detección de 2 tipos de drogas ilícitas (DI) y sus principales metabolitos en muestras de aguas residuales de cinco plantas de tratamiento de aguas residuales (PTAR) en la ciudad de Río de Janeiro. Métodos: Como clase de contaminantes de preocupación emergente, las Dls deben tenerse en cuenta debido a sus riesgos sociales y de salud pública. En este estudio, se recolectaron muestras en PTARs para evaluación per cápita de Dls mediante método de epidemiología de alcantarilla (WBE). Resultados: Las

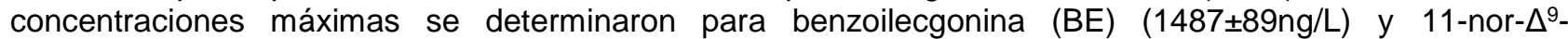
hydroxytetrahydrocannabinol (THC-COOH) $(788 \pm 27 \mathrm{ng} / \mathrm{L})$. En relación con los datos de consumo estimados se obtuvo que la sustancia más consumida por cada 1.000 habitantes (entre 15 y 65 años) fue el $\Delta^{9}$ tetrahidrocannabinol (THC) (compuesto fundamental de marihuana), con un valor de 1743,4-12422,5 mg/día, seguido de cocaína, con un valor de 987,5-1641,3 mg/día. Conclusión: El uso de drogas ilícitas contribuye sustancialmente a la carga mundial de enfermedad, morbilidad y delincuencia organizada. La aplicación del método analítico para la detección de DI brindará nuevas estrategias de monitoreo de la salud a través de la detección temprana en PTARs de estos compuestos y, fundamentalmente, la formulación de políticas públicas específicas de acuerdo con los hallazgos.
\end{abstract}

Palabras clave: Drogas ilícitas, Consumidores de drogas, Epidemiología de alcantarilla, Salud pública, Derechos humanos.

\section{RESUMO}

Objetivo: Detecção de 2 tipos de drogas ilícitas (DI) e seus principais metabólitos em amostras de esgoto coletadas em cinco estações de tratamento de esgoto (ETE) na cidade do Rio de Janeiro. Métodos: Como uma classe de poluentes de preocupação emergente, os Dls devem ser considerados devido aos seus riscos sociais e de saúde pública. Neste estudo, as amostras foram coletadas em ETEs para avaliação per capita de Dls pelo método de epidemiologia baseada em águas residuais (WBE). Resultados: As concentrações máximas foram determinadas para benzoilecgonina (BE) (1487 $\pm 89 \mathrm{ng} / \mathrm{L})$ e 11-nor- $\Delta^{9}$ hydroxytetrahydrocannabinol (THC-COOH) $(788 \pm 27 \mathrm{ng} / \mathrm{L})$. Em relação aos dados de consumo estimados, obteve-se que a substância mais consumida por 1.000 habitantes (entre 15 e 65 anos) foi o $\Delta^{9}$ tetrahidrocannabinol (THC) (composto fundamental da maconha), com valor de 1743,4-12422,5 mg/dia, seguido de cocaína, com um valor de 987,5-1641,3 mg/dia. Conclusão: $O$ uso de drogas ilícitas contribui substancialmente para o fardo global de doenças, morbidade e crime organizado. A aplicação do método analítico para detecção de DI proporcionará novas estratégias de monitoramento da saúde por meio da detecção precoce desses compostos em ETEs e, fundamentalmente, a formulação de políticas públicas específicas de acordo com os achados.

Palavras-chave: Drogas ilícitas, Usuários de drogas, Epidemiologia de esgotos, Saúde pública, Direitos humanos.

\footnotetext{
${ }^{1}$ Escola Nacional de Saúde Pública Sergio Arouca (ENSP/FIOCRUZ), Rio de Janeiro - RJ.

*E-mail: aldoferreira@ensp.fiocruz.br

${ }^{2}$ Comisión Nacional contra las Adicciones (CONADIC), Ciudad de México, México.
}

Conselho Nacional de Desenvolvimento Científico e Tecnológico (CNPq) 


\begin{abstract}
Objective: Detection of 2 types of illicit drugs (ID) and their main metabolites in wastewater samples were collected from five wastewater treatment plants (WWTP) in the city of Rio de Janeiro. Methods: As a class of pollutants of emerging concern, IDs should be considered due to their social and public health risks. In this study, samples were collected in WWTPs for per capita evaluation of IDs by wastewater-based epidemiology (WBE) method. Results: The maximum concentrations were determined for benzoylecgonine (BE) $(1487 \pm 89 \mathrm{ng} / \mathrm{L})$ and $11-$ nor- $\Delta^{9}$-hydroxytetrahydrocannabinol $(\mathrm{THC}-\mathrm{COOH})(788 \pm 27 \mathrm{ng} / \mathrm{L})$. In relation to the estimated consumption data, it was obtained that the substance most consumed per 1,000 inhabitants (between 15 and 65 years old) was $\Delta^{9}$-tetrahidrocannabinol (THC) (fundamental compound of marijuana), with a value of $1743.4-12422.5 \mathrm{mg} / \mathrm{day}$, followed by cocaine, with a value of $987.5-1641.3 \mathrm{mg} / \mathrm{day}$. Conclusion: The use of illicit drugs contributes substantially to the global burden of disease, morbidity and organized crime. The application of the analytical method for the detection of ID will provide new health monitoring strategies through the early detection of these compounds in WWTPs and, fundamentally, the formulation of specific public policies in accordance with the findings.
\end{abstract}

Keywords: Illicit drugs, Drug users, Wastewater-based epidemiology, Public health, Human rights.

\title{
INTRODUCCIÓN
}

Los psicoactivos se convirtieron en objeto de investigación socio-antropológica tardíamente (BALLANI TSL y OLIVEIRA MLF, 2007). En la primera mitad del siglo XX, lo que fue producido de forma disciplinaria, como investigación sociológica académicamente establecida, tuvo un carácter eminentemente accesorio, como forma de complementación de los esfuerzos médicos y sanitarios en entender y resolver los problemas relacionados al consumo de drogas: dependencia, crimen y disgregación social (BERNAL E, 2002).

El cannabis y la cocaína son drogas ilícitas (DI) de mayor consumo (JIMENEZ HERRERA L, 2010). La marihuana es el nombre dado a las hojas y flores secas de la planta Cannabis sativa, preparada como una mezcla para fumar; en otro estado, hachís es la resina extraída. La planta se utiliza desde hace siglos para fines recreacionales y medicinales. Contiene en promedio 400 sustancias químicas y al menos 60 alcaloides conocidos como cannabinoides. Entre ellos, el tetrahidrocannabinol (THC) es el más activo y principal responsable de los efectos producidos (RIBEIRO M, et al., 2005).

La cocaína (COC) es un alcaloide natural, aislado de las hojas de la planta Erythroxylum coca originaria de Sudamérica. Tradicionalmente, los indígenas mascaban la hoja de coca para paliar el hambre, la sed y superar los esfuerzos requeridos durante sus largas jornadas de trabajo, poniendo de manifiesto algunas de las propiedades farmacológicas de este alcaloide. La cocaína es un psicoestimulante simpaticomimético y fue el primer anestésico local que se descubrió. Es un ester benzoico y contiene una base nitrogenada con la misma estructura que los anestésicos locales que se sintetizan actualmente (FERREIRA AP, 2019).

El consumo de DI ha sido una preocupación creciente en todo el mundo. El Informe Mundial sobre las Drogas de 2018 señala que más de 275 millones de residentes en todo el mundo consumían drogas, con miles de toneladas de DI consumidas anualmente (UNODC, 2018). El consumo de drogas forma parte de un problema multidimensional, por lo que no basta con limitarlo a una relación reduccionista entre una persona y una sustancia; es necesario considerar esta interacción y sus relaciones sociales, económicas y políticas (JONES AW y HOLMGREN A, 2014).

Los estudios sobre la presencia de DI en las aguas crudas de las plantas de tratamiento de aguas residuales (PTAR) proporcionan información invaluable sobre el uso de DI y las tendencias de los consumidores a nivel local, nacional e internacional (NEFAU T, et al., 2013, GAO T, et al. 2017, JÓŻWIAKOWSKI K, et al. 2021). En última instancia, las DI ingresan al sistema de aguas residuales como formas inalteradas $\mathrm{y} / \mathrm{o}$ metabolitos. Estas drogas fueron excretadas luego del consumo humano, o fueron liberadas ilegalmente luego de la intervención policial (THOMAS KV, 2012).

Así, la epidemiología de alcantarilla (WBE) constituye una herramienta importante para la estimación del consumo local a través de la investigación de los flujos de masa de drogas de abuso inalteradas o de sus 
metabolitos. Además, se reconoce este método como una alternativa a los métodos oficiales basados en entrevistas, datos médicos y estadísticas criminales para establecer el consumo de las Dls por parte de la población local (ZUCCATO E, et al., 2008, POSTIGO C, et al., 2010, JACOX A, et al. 2017).

Las DI son de preocupación emergente para la salud pública (MOLD A, 2018). El estudio de estos contaminantes tiene un doble aspecto: epidemiológicamente, la magnitud, la naturaleza y los patrones de consumo se pueden evaluar a través de la gama de compuestos detectados en las PTAR, ambientalmente, el impacto que estas sustancias pueden representar para los ecosistemas (VAN NUIJS, et al., 2011).

Un enfoque holístico requiere múltiples aspectos, como diseñar un sistema de salud adecuado que responda a las necesidades de los consumidores de drogas, incluidos los centros de tratamiento y los servicios de rehabilitación. Para lograr estos objetivos, los responsables de la formulación de políticas requieren datos de consumo racionales, realistas, basados en pruebas y en tiempo real. Por esta razón, en las últimas décadas se adoptó un enfoque de indicadores múltiples para una estimación más precisa del consumo de drogas a nivel comunitario. Este enfoque de indicadores múltiples incluye herramientas de apoyo adicionales como la WBE para llenar los vacíos (como sesgos y retrasos) de otros métodos convencionales (EMCDDA, 2016), bien como restituir la dignidad basada en los derechos humanos (CARPIZO J, 2011).

De esa forma, se constituye un enfoque alternativo para estimar la escala del consumo de drogas dentro de una comunidad. Los datos generados por este método proporcionan la cantidad total de cada droga utilizado dentro de una comunidad, comúnmente expresada como dosis por día o semana por cada 1000 personas (DAUGHTON CG, 2001, ZUCCATO E, et al., 2008). Por su naturaleza, esto es una función tanto del número de personas que consumen una droga como de la frecuencia de uso.

Mientras que el porcentaje de la población que consume cada droga es una medida muy importante, el grado de uso de cualquier droga en particular en una comunidad y, por lo tanto, la carga de enfermedad, el crimen y otros costos relacionados con las drogas, también están determinados por la frecuencia con la que se consume usado. Las concentraciones de drogas en las aguas residuales suelen oscilar entre unas pocas unidades y cientos de nanogramos por litro (NEFAU T, et al., 2013, JACOX A, et al. 2017). Como en otros países o regiones, el tipo de drogas y sus concentraciones en los efluentes pueden variar considerablemente según la región.

Los datos disponibles en la literatura, que es representativo de la población en su conjunto, informan categorías de frecuencia de uso, como "diario", "semanal", "mensual" y "una o dos veces al año" (REID MJ, et al., 2012). Sin embargo, estas categorías dificultan la estimación de la cantidad total de cada droga consumida en la comunidad.

Degenhardt L y Hall W (2012) han señalado que incluso las tasas de dependencia en comparación con el uso ocasional de diferentes DI son datos que no están ampliamente disponibles dadas las fuentes actuales. Tal detalle puede ser difícil de lograr para las encuestas si la recopilación de los encuestados sobre la pureza, las cantidades y la frecuencia utilizadas es inexacta o imprecisa. Los usuarios habituales también pueden ser difíciles de alcanzar en este tipo de encuestas. Otra limitación es el lapso de tiempo entre encuestas, lo que significa que los cambios de uso a corto plazo no se reflejan.

Así, el objetivo de este estudio fue investigar el uso de 2 tipos de DI y sus principales metabolitos, utilizando análisis de aguas residuales.

\section{MÉTODOS}

Para recolección de muestras, los datos del caudal de las Planta de Tratamiento de Aguas Residuales (PTAR) analizadas: PTAR Alegría (5,000 L/s), PTAR Penha (1,086 L/s), PTAR llha do Governador (525 L/s), PTAR Pavuna $(1,500 \mathrm{~L} / \mathrm{s})$ y PTAR Sarapui $(1,500 \mathrm{~L} / \mathrm{s})$. La recolección de muestras siguió las orientaciones establecidas por Ort C, et al. (2010) y Castiglioni S, et al. (2013). Las campañas de muestreo fueran hechas a través de técnica de muestreo-composta en septiembre/2018. Se tomaron $1000 \mathrm{~mL}$ de muestra en botellas de vidrio borisilicatado ámbar. Las muestras se mantuvieron entre 2-5C (Figura 1). 
Figura 1 - Área de estudio: Plantas de tratamiento de aguas residuales analizadas. Rio de Janeiro, 2021.
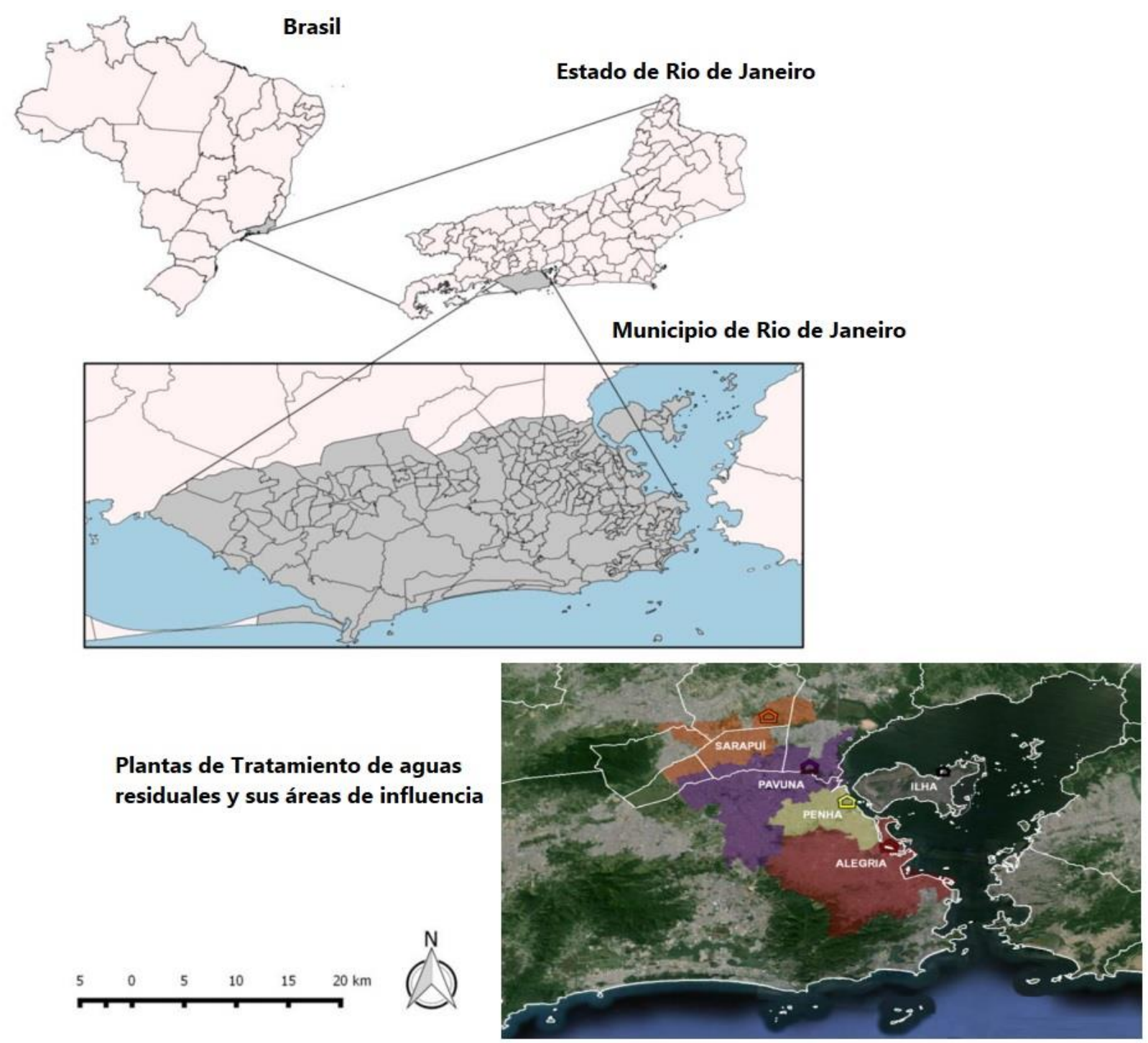

Fuente: Ferreira AP y Cruz-Hernández MJ (2021) adaptado de CEDAE (2021).

Soluciones estándar de Cocaína (COC), Benzoilecgonina (BE), Nor-benzoilecgonina (Nor-BE), Éster metílico de ecgonina (EME), Cocaetileno (CE), $\Delta^{9}$-tetrahidrocannabinol (THC), 11-nor-9-carboxi- $\Delta^{9}$ tetrahidrocannabinol (THC-COOH), 11-hidroxi- $\Delta^{9}$-tetrahidrocannabiol $(\mathrm{OH}-\mathrm{THC})$, y análogos deuterados $\left(\mathrm{COC}-\mathrm{d}_{3}, \mathrm{BE}-\mathrm{d}_{3}, \mathrm{EME}-\mathrm{d}_{3}, \mathrm{CE}-\mathrm{d}_{8}, \mathrm{THC}-\mathrm{COOH}-\mathrm{d}_{3}\right)$, en metanol $(\mathrm{MeOH}) \mathrm{o}$ acetonitrilo (ACN), adquirido de Cerilliant (Round Rock, Texas, EUA). MeOH y ACN (Hipersolv Chromanorm), ácido fórmico (AF) (Normapur) adquirido de J.T. Baker (Xalostoc, México). Agua ultrapura fue producida usando sucesivas filtraciones por ósmosis inversa Milli-RO y sistema de purificación de agua Milli-Q Plus (Millipore, MA, USA). Para extracción en fase sólida (SPE) columna Pursuit $C_{18}(10 \mu \mathrm{m}, 20 \times 2.0 \mathrm{~mm})$ y Xbridge Phenyl $3.5 \mathrm{~mm}, 3 \mathrm{~mm} \times 150 \mathrm{~mm}$ columna HPLC (HLB Oasis, Waters, Milford, EUA).

El análisis de las muestras de agua residual se realizó en el laboratorio de la Universitat Jaume I de Castellón (UJI). Se analizaron 2 drogas y 6 metabolitos pertenecientes a cinco clases diferentes de compuestos químicos similares a los cannabinoides y a los cocaínicos. Los cannabinoides son el principal componente psicoactivo de la planta de marihuana, formándose THC y dos productos metabólicos, THC$\mathrm{COOH}$ y $\mathrm{OH}-\mathrm{THC}$. Dentro del grupo de los cocaínicos se incluye la COC, su principal metabolito (BE), en menor proporción (Nor-BE) y en caso de que se produzca transesterificación entre la COC y el etanol se forma CE. La Tabla 1 presenta los límites de detección de cada compuesto. 
Tabla 1 - Drogas ilícitas y metabolitos analizados, abreviaturas, fórmulas, números CAS, límites de detección y factores de corrección. Rio de Janeiro, 2021.

\begin{tabular}{|c|c|c|c|c|c|}
\hline \multicolumn{2}{|c|}{$\begin{array}{c}\text { Grupos } \\
\text { de } \\
\text { drogas }\end{array}$} & \multirow{2}{*}{$\begin{array}{l}\text { Abreviaturas } \\
\text { THC }\end{array}$} & \multirow{2}{*}{$\begin{array}{c}\begin{array}{c}\text { Números } \\
\text { CAS* }\end{array} \\
1972-08-3\end{array}$} & \multirow{2}{*}{$\begin{array}{c}\begin{array}{c}\text { Límites } \\
\text { de } \\
\text { detección } \\
(\mathbf{n g} / \mathrm{L})\end{array} \\
5\end{array}$} & \multirow{2}{*}{$\begin{array}{r}\begin{array}{c}\text { Factor de } \\
\text { corrección }\end{array} \\
2,04\end{array}$} \\
\hline $\mathscr{\&}$ & $\Delta^{9}$-tetrahidrocannabinol & & & & \\
\hline$\frac{\mathrm{E}}{0}$ & 11-hidroxi- $\Delta^{9}$-tetrahidrocannabiol & $\mathrm{OH}-\mathrm{THC}$ & $36557-05-8$ & 2,5 & 0,37 \\
\hline ల్ల & 11-nor-9-carboxi- $\Delta^{9}$-tetrahidrocannabinol & $\mathrm{THC}-\mathrm{COOH}$ & $23978-85-0$ & 60 & 1,26 \\
\hline \multirow{4}{*}{ 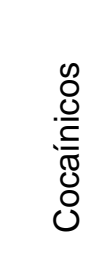 } & Benzoilecgonina & $\mathrm{BE}$ & $519-09-5$ & 10 & 2,3313 \\
\hline & Cocaína & $\operatorname{coc}$ & $50-36-2$ & 2,5 & - \\
\hline & Nor-Benzoilecgonina & Nor-BE & $(-)$ & 2,5 & 3,71 \\
\hline & Cocaetileno & CE & $529-38-4$ & 5 & 4,781 \\
\hline
\end{tabular}

Nota: *Los números CAS son identificadores numéricos asignados a cada compuesto químico descrito en la literatura. Esta asignación la realiza una división de la Sociedad Americana de Química, Chemical Abstracts Service (CAS). (-): No existe número CAS. -: No aplica.

Fuente: Ferreira AP y Cruz-Hernández MJ (2021).

Las muestras fueron filtradas en vacío a través de filtros de fibra de vidrio de $1 \mu \mathrm{m}$ y filtros de membrana de nilón de 0,45 $\mu \mathrm{m}$ (Whatman International Ltd., Maidstone, Inglaterra). Posteriormente, se almacenaron en recipientes de ámbar polietileno tereftalato a una temperatura de menos $20^{\circ} \mathrm{C}$ en total oscuridad hasta su posterior análisis. Se utilizaron patrones de solución de alta pureza de los compuestos de destino y sus análogos deuterados (Cerilliant, Round Rock, Texas, EUA) como solución en MeOH o ACN. La metodología aplicada para el análisis de drogas y sus metabolitos se realizaron según Senta I, et al. (2013).

La estimación del consumo de drogas consistió en obtener la cantidad de residuo de droga por día (ng/día) multiplicando la concentración de la droga o su metabolito expresado en $\mathrm{ng} / \mathrm{L}$ por la tasa de flujo de agua que ingresó a la PTAR el día del muestreo (L/día). La cantidad de residuo de cada droga se ajustó utilizando un factor de corrección (POSTIGO C, et al., 2008).

Los estudios epidemiológicos de las aguas residuales se basan en los niveles de las DI y sus metabolitos en los efluentes de las PTARs teniendo en cuenta algunos factores farmacocinéticos y metabólicos, así como el destino de los productos de excreción. Un esquema general del análisis de las aguas residuales enfocado a determinar el consumo de drogas de abuso entre una población se muestra en la Figura 2.

El primer paso consiste en recolectar una muestra representativa del agua de entrada de la PTAR para su posterior análisis cuantitativo (paso 2). Este paso es crucial para obtener una cuantificación exacta (concentración en $\mathrm{ng} / \mathrm{L}$ ) de cada droga residual. En los pasos 3-5 se muestran los parámetros necesarios para estimar el consumo de la sustancia en determinada población que generalmente se expresa en $\mathrm{mg} / \mathrm{día} / 1000$ habitantes.

Estos parámetros son la concentración hallada en el paso 2, el caudal del agua de entrada, el número de población servida por la PTAR entre 15 y 65 años y el factor de corrección usado para cada droga, el cual es obtenido considerando la tasa de excreción media de la sustancia/metabolito y la tasa del peso molecular de la sustancia/metabolito. El uso de los metabolitos en los cálculos es preferible sobre el compuesto precursor porque los metabolitos son una prueba de consumo humano, mientras que el compuesto precursor también puede estar presente en las aguas residuales debido a la eliminación deliberada de la sustancia. 
Figura 2 - Esquema general en epidemiología de alcantarilla. Rio de Janeiro, 2021.

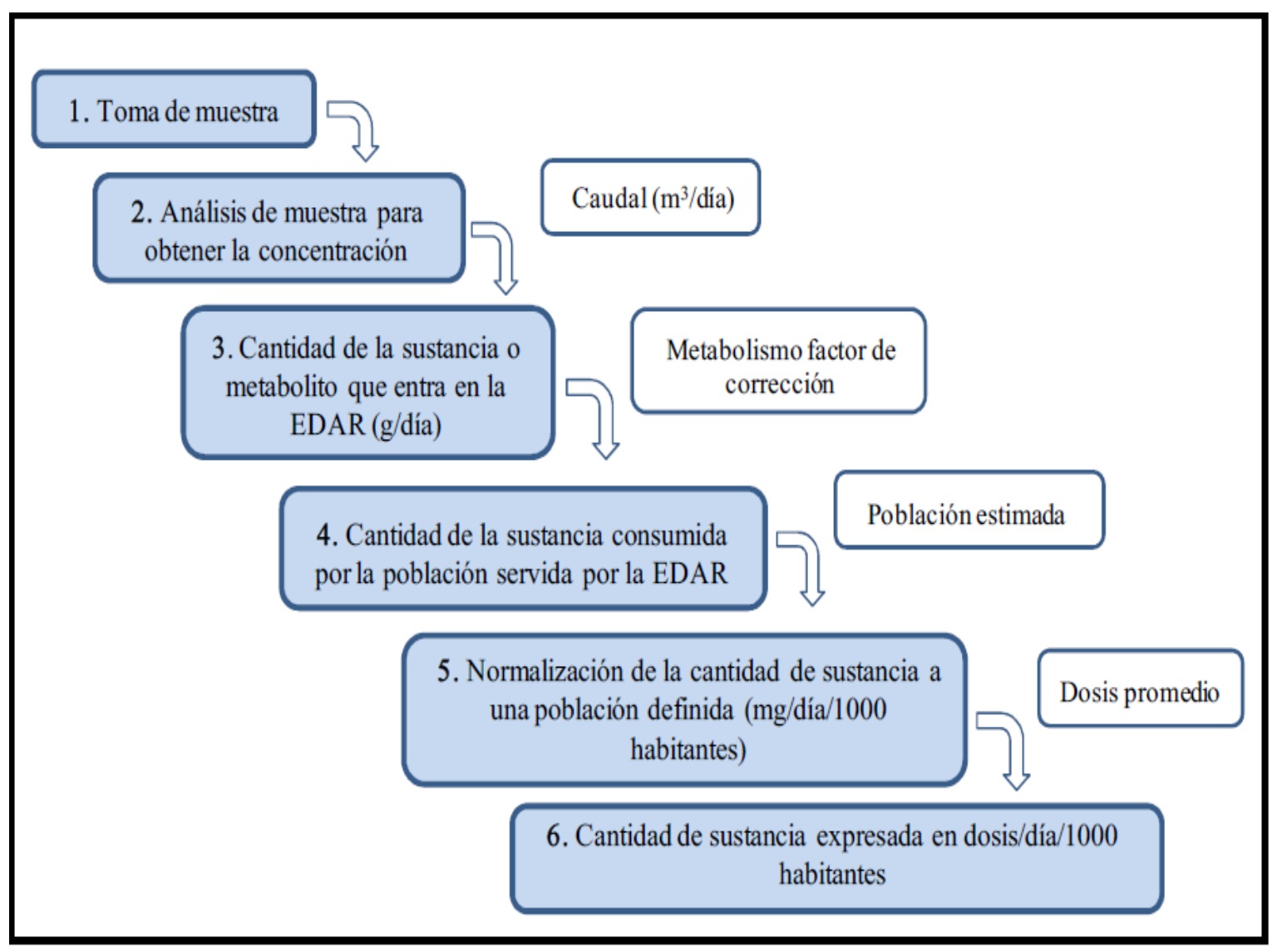

Fuente: Ferreira AP y Cruz-Hernández MJ (2021).

La concentración de las drogas y sus metabolitos fue descrita utilizando concentración media por sitio de muestreo. La estimación del consumo de cocaína y marihuana se vinculó con la ubicación de las PTAR participantes del estudio.

La estimación del consumo de drogas en aguas residuales proporciona información sobre la cantidad y tipo de drogas consumidas en una ciudad. Para aquellos productos de excreción que son estables en agua residual y que, por tanto, llegan prácticamente intactos a la PTAR, es razonable asumir que la cantidad excretada de forma colectiva se vea reflejada por la carga total recibida en la PTAR en un determinado intervalo de tiempo. La recogida de muestras representativas para dicho intervalo temporal es, en consecuencia, uno de los factores clave en este enfoque (POSTIGO C, et al., 2008).

Este método se basa en primer lugar, en asumir que las drogas después de que son consumidas y metabolizadas en el cuerpo humano son excretadas como compuestos principales/metabolitos y pueden llegar a las PTAR a través de las aguas residuales procedentes de los sanitarios. En segundo lugar, se basa en que son conocidas las rutas metabólicas y principales metabolitos de las drogas y fármacos. Por último, se asume que la cantidad de droga o metabolito cuantificado en aguas residuales corresponde con la dosis consumida. Los parámetros necesarios para poder calcular el uso de drogas incluyen la concentración de droga en las aguas residuales, la población a la que sirve la PTAR, los metabolitos y rutas metabólicas de las drogas analizadas, es decir, el porcentaje de droga o principal metabolito excretado.

El análisis de datos fue realizado con el programa estadístico STATA versión 13 (StataCorp LP) y los gráficos realizados con el programa $\mathrm{R}$, versión 3.1.0.

El presente trabajo contó con la aprobación del Comité de Ética en Investigación Científica de la Escola Nacional de Saúde Pública Sergio Arouca (Ensp/Fiocruz), bajo el Dictamen 07/2018. 


\section{RESULTADOS}

Los análisis de aguas residuales municipales mostraron una presencia ubicua de los compuestos investigados en las PTAR seleccionadas con concentraciones individuales que varían ampliamente. Casi todas las muestras analizadas (99\%-100\%) contenían concentraciones medibles de BE, COC y THC-COOH. La Tabla 2 presenta la media de la concentración de DI y metabolitos (ng/L) en las muestras recolectadas (1PTAR Alegria, 2- PTAR Penha, 3- PTAR Ilha do Governador, 4- PTAR Pavuna y 5- PTAR Sarapui) y la Tabla 3 presenta la estimación del consumo diario de Marihuana y Cocaina.

Tabla 2 - Media de la concentración de drogas ilícitas y metabolitos ( $\mathrm{ng} / \mathrm{L}$ ) en las muestras recolectadas (1PTAR Alegria, 2- PTAR Penha, 3- PTAR Ilha do Governador, 4- PTAR Pavuna y 5- PTAR Sarapui).

Rio de Janeiro, 2021.

\begin{tabular}{|c|c|c|c|c|c|c|}
\hline \multirow{2}{*}{\multicolumn{2}{|c|}{ Droga y metabolitos }} & \multicolumn{5}{|c|}{ Concentración (ng/L) } \\
\hline & & PTAR-1 & PTAR-2 & PTAR-3 & PTAR-4 & PTAR-5 \\
\hline \multirow{3}{*}{ 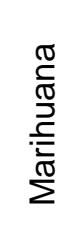 } & THC & - & - & - & - & - \\
\hline & $\mathrm{OH}-\mathrm{THC}$ & - & 4,7 & - & 2,4 & - \\
\hline & $\mathrm{THC}-\mathrm{COOH}$ & 463 & 310 & 788 & 353 & 268 \\
\hline \multirow{4}{*}{ 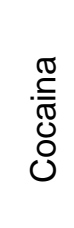 } & $\mathrm{BE}$ & 680 & 533 & 440 & 1487 & 692 \\
\hline & $\mathrm{COC}$ & 334 & 323 & 208 & 482 & 220 \\
\hline & Nor-BE & - & 19,6 & - & - & - \\
\hline & CE & - & - & - & - & - \\
\hline
\end{tabular}

Fuente: Ferreira AP y Cruz-Hernández MJ (2021).

Tabla 3 - Cuantificación del consumo de Marihuana y Cocaína (mg/día por cada 1000 habitantes) referente a la populación de las regiones estudiadas. Rio de Janeiro, 2021.

\begin{tabular}{|c|c|c|c|c|c|}
\hline \multirow{2}{*}{ Droga } & \multicolumn{5}{|c|}{$\begin{array}{l}\text { Consumo de drogas } \\
\text { (mg/día por cada } 1000 \text { habitantes) }\end{array}$} \\
\hline & PTAR-1 & PTAR-2 & PTAR-3 & PTAR-4 & PTAR-5 \\
\hline 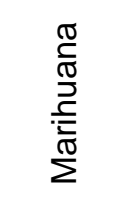 & 4163,2 & 12422,5 & 1935,0 & 1979,6 & 1743,4 \\
\hline 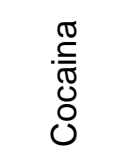 & 1641,3 & 1181,7 & 1332,6 & 987,5 & 1285,8 \\
\hline
\end{tabular}


Con relación a la determinación de DI en aguas residuales, la BE apareció en todos los puntos analizados, coincidiendo con los resultados reportados por Postigo C, et al. (2008). Fue detectado Nor-BE y cocaetileno (metabolitos de la cocaína), con valores similares presentados de otros estudios realizados (KASPRZYKHORDERN B, et al., 2009, JONES AW y HOLMGREN A, 2014. Las concentraciones medias más altas se determinaron para $\mathrm{BE}(1487 \pm 89 \mathrm{ng} / \mathrm{L})$ y $\mathrm{THC}-\mathrm{COOH}(788 \pm 27 \mathrm{ng} / \mathrm{L})$, mientras que relativamente baja y rara se determinaron para Nor-BE $(19,6 \pm 8 \mathrm{ng} / \mathrm{L})$ y OH-THC $(4,7 \pm 0,5 \mathrm{ng} / \mathrm{L})$.

En relación con los datos estimados de consumo se obtuvo que la sustancia más consumida por cada 1000 habitantes fue el THC (compuesto fundamental de la marihuana), con un valor de 1743,4-12422,5 miligramos/día, seguido por la cocaína con un valor de 987,5-1641,3 miligramos/día. El consumo de drogas estimado a partir de las concentraciones de influente indica que la población consumió principalmente marihuana, seguido de cocaína.

\section{DISCUSIÓN}

El consumo de drogas es una nueva preocupación que debe considerarse no solo para los aspectos sociales y de salud pública, sino también en un contexto ambiental para la contaminación de las aguas superficiales (MOLD A, 2018). Como se muestra en la Tabla 1, a partir de la concentración de BE en el alcantarillado doméstico es posible realizar una estimación del consumo de cocaína por la población servida por la PTAR en estudio. Este enfoque fue conceptualmente desarrollado por Daughton CG (2001).

Fue observado la presencia del THC-COOH, BE y COC en todos los análisis. En este estudio se detectó OH-THC en la PTAR-Pavuna (2,4 ng/L) que es similar a la encontrada en Talavera de la Reina y en el Río Tajo (CHICHARRO AF, et al., 2014). Cabe destacar que la ausencia de esta sustancia en la mayoría de las muestras analizadas puede deberse a que solo $20 \%$ de marihuana consumida es excretado por la orina y se elimina prioritariamente en forma del principal metabolito $\mathrm{THC}-\mathrm{COOH}$, seguido del OH-THC (FERREIRA AP, 2019).

Los datos encontrados en esta investigación son preocupantes, ya que son similares a los presentados en regiones con mayor consumo, como lo demuestran los resultados señalados por UNODC (2018), comparativos con el consumo de DI en países europeos: Suiza en Zuccato E, et al. (2008), Reino Unido en Kasprzyk-Hordern B, et al. (2009), en 19 ciudades europeas según Thomas KV, et al. (2012), Francia en Nefau T, et al. (2013), Suecia en Ostman M, et al. (2014), España en Bijlsma L, et al. (2018) y Italia en Cosenza A, et al. (2018); Australia según Lai FY, et al. (2013); América del Norte en Jones-Lepp TL, et al. (2004), Bartelt-Hunt SL, et al. (2009), Metcalfe C, et al. (2010) y algunos en América del Sur: Colombia en Bijlsma L, et al. (2016) y Brasil en Silva KM, et al. (2018).

El consumo es independiente del poder adquisitivo de la población, pues a pesar de la vigilancia policial, el tráfico siempre busca cada vez más poner a disposición una gran cantidad de droga (EMCDDA, 2016). Con eso, siempre hay disponibilidad de DI, que es una tragedia para la sociedad, para la salud pública, lo que impacta significativamente cualquier acción exitosa en las políticas públicas de prevención.

Otro punto importante por destacar es la razón (COC/BE). Por ser un producto de excreción es muy baja la proporción de $\mathrm{COC}$ sin biotransformar y lógicamente, la BE es más abundante llegando a ser hasta 100 veces más que la COC. Los resultados fueran: 0,49 (PTAR-1), 0,60 (PTAR-2), 0,47 (PTAR-3), 0,32 (PTAR-4) y 0,32 (PTAR-5). Según Kasprzyk-Hordern B, et al. (2009), esta razón puede deducir si los compuestos presentes en el alcantarillado provienen del consumo de cocaína o del aporte de la droga antes de ser consumida.

En general, la razón COC/BE menor que 0,27 indica el aporte de cocaína consumida, a pesar de existir autores que trabajan con una razón límite de 0,75 . Por lo tanto, desde el punto de vista forense, la razón $\mathrm{COC} / \mathrm{BE}$ es fundamental para la identificación del aporte de drogas in natura en el sistema de alcantarillado sanitario pudiendo ser utilizada por las fuerzas de inteligencia policial como una herramienta de localización de laboratorios de refino o manejo de esas sustancias.

Según Bijlsma L, et al. (2018), para poder evaluar el impacto de las intervenciones dirigidas a reducir el consumo de drogas, los legisladores precisan de información rigurosa y veraz, de forma que consigan 
establecer sus decisiones en evidencias científicas. Tradicionalmente, el consumo de drogas se ha estimado a partir de encuestas poblacionales y otros indicadores como cantidades de droga incautadas por la policía, crímenes y defunciones relacionadas con drogas, registros hospitalarios, o personas en tratamiento de drogadependencia.

Una dificultad común de estas metodologías es que los procesos implicados en la obtención de información son prolongados en términos de ejecución, caros y propensos a estar sesgados por el tabú social que supone reconocer el consumo de este tipo de sustancias (NEFAU T, et al., 2013). Además, la información obtenida depende en gran medida de la eficacia policial para combatir el narcotráfico. Así pues, es fundamental impulsar el desarrollo de nuevos enfoques, complementarios a los tradicionales, que permitan alcanzar datos de forma objetiva, rápida, cuantitativa y fiable.

El consumo de DI en la sociedad es actualmente uno de los mayores problemas sociales existentes y está surgiendo también como un posible problema ambiental. La presencia de DI en matrices ambientales, principalmente aguas superficiales, surge como una cuestión asociada al aporte de efluentes de PTAR y, en países con bajo índice de tratamiento de aguas residuales como Brasil, está asociada al aporte de alcantarillado de aguas servidas. Por consiguiente, debido a la complejidad de las matrices y la importancia de la determinación de estas sustancias, el desarrollo de métodos analíticos fiables es una necesidad real.

Es necesario realizar evaluaciones oportunas para que los países que pudieran verse afectados por el aumento del tráfico comprendan la magnitud de las corrientes de tráfico y se equipen debidamente de modo que puedan prestar servicios para prevenir la expansión del consumo de drogas y dispensar tratamiento y servicios a fin de reducir al mínimo las consecuencias adversas que el consumo de drogas puede entrañar para la salud.

\section{CONCLUSIÓN}

El concepto de epidemiología de alcantarilla utiliza las concentraciones medidas de DI en las PTAR centralizadas para volver a calcular el uso de drogas en las comunidades locales. De esa forma, el estudio de monitorización llevado a cabo en cinco PTAR localizadas en diferentes zonas geográficas, aporta información relevante sobre la presencia y concentraciones consumidas de las DI. Las ubicaciones de muestreo se distribuyeron en las principales PTAR y se tomó especial cuidado para seleccionarlas de varios tamaños para resaltar las tendencias del consumo local de drogas y compararlas con otros estudios. Demuestra la relevancia del método de la epidemiología de alcantarilla que debe considerarse como una herramienta eficiente para apoyar las actividades de reducción de daños y la política de prevención de salud pública. Proporciona a las autoridades, estadísticas de tiempo casi real, para identificar las tendencias y tasas de uso, los puntos de consumo y la efectividad de las contramedidas.

\section{FINANCIAMENTO}

El estudio fue financiado por el Conselho Nacional de Desenvolvimento Científico e Tecnológico (CNPq).

\section{REFERENCIAS}

1. BALLANI TSL, OLIVEIRA MLF. Use of drugs of abuse and sentinel event: constructing a proposition about assessing public policies. Texto Contexto Enfermagem. 2007; 16(3):488-494.

2. BARTELT-HUNT SL, et al. The occurrence of illicit and therapeutic pharmaceuticals in wastewater effluent and surface waters in Nebraska. Environmental Pollution. 2009; 157:786e791.

3. BERNAL E. ¿Tienen futuro las guías de práctica clínica en el Sistema Nacional de Salud? Gestión Clínica y Sanitaria. 2002; 4(3):75-77.

4. BIJLSMA L, et al. Análisis de aguas residuales con fines epidemiológicos: aplicaciones a la estimación del consumo de sustancias de abuso y en salud pública en general. Revista Española de Salud Pública. 2018; 92:e201808053.

5. BIJLSMA L, et al. Estimation of illicit drug use in the main cities of Colombia by means of urban wastewater analysis. Science of the Total Environment. 2016; 565:984-993.

6. CARPIZO J. Los derechos humanos: naturaleza, denominación y características. Revista Cuestiones Constitucionales. 2011; 25:3-29.

7. CASTIGLIONI S, et al. Evaluation of uncertainties associated with the determination of community drug use through the measurement of sewage drug biomarkers. Environmental Science \& Technology. 2013; 47(3):1452-1460. 
8. CEDAE. Companhia Estadual de Água e Esgotos. Disponível em: https://www.cedae.com.br/tratamento_esgoto/tipo/historia-do-tratamento-de-esgoto. Acesso em: 20 fevereiro 2020.

9. CHICHARRO AF, et al. Estimación del consumo de drogas de abuso y sus metabolitos a partir de su presencia en el agua residual de Talavera de la Reina y en el Río Tajo. Revista Española de Salud Pública. 2014; 88(2):289-299.

10. COSENZA A, et al. Maida CM, Piscionieri D, Fanara S, Di Gaudio F, Viviani G. Occurrence of illicit drugs in two wastewater treatment plants in the South of Italy. Chemosphere. 2018; 198:377e385.

11. DAUGHTON CG. Illicit Drugs in Municipal Sewage. Pharmaceuticals and Care Products in the Environment. Washington, DC: American Chemical Society, 2001.

12. DEGENHARDT L, HALL W. Extent of illicit drug use and dependence, and their contribution to the global burden of disease. Lancet. 2012; 379:55-70.

13. EMCDDA. Assessing illicit drugs in wastewater: advances in wastewater-based drug epidemiology, Insights 22.2016. Publications Office of the European Union, Luxembourg. Disponível em: https://doi.org/10.2810/017397. Acesso em: 21 de junho de 2020.

14. FERREIRA AP. Estimaciones del consumo de drogas ilícitas derivadas del análisis de aguas residuales: Una revisión crítica. Revista Universidad Industrial de Santander Salud. 2019; 51(1):69-80.

15. GAO T, et al. Occurrence of new psychoactive substances in wastewater of major Chinese cities. Science of the Total Environment. 2017; 575:963-969.

16. JACOX A, et al. Quantitative analysis of opioids and cannabinoids in wastewater samples, Forensic Sciences Research 2017; 2(1): 18-25.

17. JIMENEZ HERRERA L. Prevention against drugs. Revista costarricense de salud publica. 2010; 19(2):93-100.

18. JONES AW, HOLMGREN A. Concentrations of cocaine and benzoylecgonine in femoral blood from cocaine-related deaths compared with venous blood from impaired drivers. Journal of Analytical Toxicology. 2014; 38(1):46-51.

19. JONES-LEPP TL, et al. Polar organic chemical integrative sampling and liquid chromatography electro spray ion-trap mass spectrometry for assessing selected prescription and illicit drugs in treated sewage effluents. Archives of Environmental Contamination and Toxicology. 2004; 47:427e439.

20. JÓŻWIAKOWSKI K, et al. The Influence of Household Wastewater Treatment Plants with Drainage System on the Quality of Groundwater in the Lublin Province, Poland. Journal of Ecological Engineering. 2021;22(3):18-39.

21. KASPRZYK-HORDERN B, et al. Illicit drugs and pharmaceuticals in the environment--forensic applications of environmental data. Part 1: estimation of the usage of drugs in local communities. Environmental Pollution. 2009; 157:1773e1777.

22. LAI FY, et al. Using quantitative wastewater analysis to measure daily usage of conventional and emerging illicit drugs at an annual music festival. Drug and Alcohol Review. 2013; 32:594e602.

23. LÓPEZ-GARCÍA E, et al. Drugs of abuse and their metabolites in river sediments: Analysis, occurrence in four Spanish river basins and environmental risk assessment. Journal of Hazardous Materials. 2020; S0304-3894(20):31301-7.

24. METCALFE C, et al. Illicit drugs in Canadian municipal wastewater and estimates of community drug use. Environmental Pollution. 2010; 158:3179e3185.

25. MOLD A. Framing drug and alcohol use as a public health problem in Britain: Past and present. Nordic Studies on Alcohol and Drugs. 2018; 35(2):93-99.

26. NEFAU T, et al. Presence of illicit drugs and metabolites in influents and effluents of 25 sewage water treatment plants and map of drug consumption in France. Science of the Total Environment. 2013; 461-462:712-722.

27. ORT C, et al. Sampling for pharmaceuticals and personal care products (PPCPs) and illicit drugs in wastewater systems: are your conclusions valid? A Critical Review. Environmental Science \& Technology. 2010; 44:6024-6035.

28. OSTMAN M, et al. A snapshot of illicit drug use in Sweden acquired through sewage water analysis. Science of the Total Environment. 2014; 472:862e871.

29. POSTIGO C, et al. Analysis of drugs of abuse and their human metabolites in water by LC-MS: A non-intrusive tool for drug abuse estimation at the community level. Trends in Analytical Chemistry. 2008; 27:1053-1069.

30. POSTIGO C, et al. Drugs of abuse and their metabolites in the Ebro River basin: occurrence in sewage and surface water, sewage treatment plants removal efficiency, and collective drug usage estimation. Environment International. 2010; 36(1):75-84.

31. REID MJ, et al. Estimation of cocaine consumption in the community: a critical comparison of the results from three complimentary techniques. BMJ Open. 2012; 2(6):e001637.

32. RIBEIRO M, et al. Abuso e dependência da maconha. Rev. Assoc. Med. Bras. 2005; 51(5):247-249.

33. SENTA I, et al. Integrated procedure for multiresidue analysis of dissolved and particulate drugs in municipal wastewater by liquid chromatography-tandem mass spectrometry. Analytical and Bioanalytical Chemistry. 2013; 405(10):3255-3268.

34. SILVA KM, et al. Assessing cocaine use patterns in the Brazilian capital by wastewater-based epidemiology. International Journal of Environmental Analytical Chemistry. 2018; 95(15):1370-1387.

35. THOMAS KV, et al. Comparing illicit drug use in 19 European cities through sewage analysis. Science of the Total Environment. 2012; 432:432-439.

36. UNODC. Global overview of drug demand and supply. 2018. Disponível em: https://www.unilibrary.org/content/books/9789210450584c003. Acesso em: 21 de junho de 2020.

37. VAN NUIJS AL, et al. Illicit drug consumption estimations derived from wastewater analysis: A critical review. Science of the Total Environment. 2011; 409(19):3564-3577.

38. ZUCCATO E, et al. Estimating community drug abuse by wastewater analysis. Environmental Health Perspectives. 2008; 116:1027-1032. 